\title{
ÍNDICE CUMULATIVO DE ARTIGOS PUBLICADOS NA RODRIGUÉSIA 2001-2005 SUPLEMENTO AO ÍNDICE CUMULATIVo 1935-2000
}

A presente listagem de artigos científicos pretende divulgar a trajetória da revista Rodriguésia, recuperando o conhecimento adquirido e armazenado em seus 70 anos de existência, formando um índice cumulativo

Armelin, Renato Soares \& Mantovani, Waldir. Definições de clareira natural e suas implicações no estudo da dinâmica sucessional em florestas. v. 52, n. 81, p. 5-15. 2001. il.

Assis, Marta Camargo de. Alstroemeriaceae no estado do Rio de Janeiro. v. 55, n. 85, p. 5-15. 2004. il.

Azevedo, Michaele Alvim Milward de \& Baumgratz, José Fernando. Passiflora L. subgênero Decaloba (DC) Rchb. (Passifloraceae) na Região Sudeste do Brasil. v. 55, n. 85, p. 17-54. 2004. il.

Biondo, Elaine; Nascimento, André R. T; Miotto, Sílvia T. S. \& Wittmann, Maria T. S. Primeiros estudos citotaxonômicos e distribuição geográfica de Rhynchosia naineckensis Fortunato (Leguminosae) para o estado de Goiás. v. 54, n. 83, p. 511.2003. il.

Bovini, Massimo G.; Carvalho-Okano, Rita Maria de \& Vieira, Milene Faria. Malvaceae A. Juss. no Parque Estadual do Rio Doce, Minas Gerais, Brasil. v. 52, n. 81, p. 17-47. 2001. il.

Caixinhas, Maria Lisete \& Liberato, Maria Cândida. Colecções botânicas do Brasil em espaços verdes notáveis de Lisboa. v. 53, n. 82, p. 25-32. 2002.

Callado, Cátia Henriques \& Silva Neto, Sebastião José da. Anatomia do lenho de três espécies do gênero Simira Aubl. (Rubiaceae) da floresta atlântica no estado do Rio de Janeiro. v. 54, n. 83, p. 23-33. 2003. il.

Carvalho, Lúcia d'Ávila Freire de; Costa, Lucio Heron P. \& Duarte, Aline Castelar. apresentado em ordem alfabética de autores. Trata-se da continuidade ao Índice Cumulativo 1935-2000, impresso na revista Rodriguésia v. 51, n. $78 / 79$ de 2000.

Diversidade taxonômica e distribuição geográfica das Solanáceas que ocorrem no Sudeste brasileiro (Acnistus, Athenaea, Aureliana, Brunfelsia e Cyphomandra). v. 52, n. 80, p. 31-45. 2001. il.

Chiquieri, Abner; Maio, Fernando Régis di \& Peixoto, Ariane Luna. A distribuição geográfica da família Rubiaceae Juss. na Flora brasiliensis de Martius. v. 55, n. 84, p. 47-57. 2004.

Costa, Maria Auxiliadora S. \& Prado, Jefferson. Flora da Reserva Ducke, Amazonas, Brasil: Pteridophyta-Metaxyaceae. v. 56, n. 86 , p. 74-75. 2005. il.

Flora da Reserva Ducke, Amazonas, Brasil: Pteridophyta - Ophioglossaceae. v. 56, n. 86, p. 72-73. 2005. il.

Esteves, Gerleni Lopes. Flora da Reserva Ducke, Amazonas, Brasil: Bombacaceae. v. 56, n. 86, p. 115-124. 2005.

Forzza, Rafaela Campostrini \& Costa, Maria Auxiliadora S. Flora da Reserva Ducke, Amazonas, Brasil: Rapateaceae. v. 56, n. 86, p. 177-181. 2005. il.

Fraga, Cláudio Nicoletti de \& Peixoto, Ariane Luna. Florística e ecologia das Orchidaceae das restingas do estado do Espírito Santo. v. 55, n. 84, p. 5-20. 2004.

Freitas, Carlos A.A \& Prado Jefferson. Flora da Reserva Ducke, Amazonas, Brasil: Pteridophyta - Dryopteridaceae. v. 56, n. 86, p. 49-52. 2005. il. \& Windisch, Paulo G. Flora da Reserva Ducke, Amazonas, Brasil: Pteridophyta - Lycopodiaceae. v. 56, n. 86, p. 67-68. 2005. 
Hopkins, Michael J.G. Flora da Reserva Ducke, Amazonas, Brasil. v. 56, n. 86, p. 9-25. 2005.

Guimarães, Elsie Franklin. Novos sinônimos para espécies de Schultesia Mart. e Xestaea Griseb. (Gentianaceae). v. 55, n. 85, p. 67-72. 2004.

Guimarães, Elsie Franklin; Giordano, Luiz Carlos da Silva. Piperaceae no Nordeste brasileiro I: estado do Ceará. v. 55, n. 84, p. 21-46. 2004. il.

Jung-Mendaçolli, Sigrid L. \& Bernacci, Luís Carlos. Myrsinaceae da APA de Cairuçu, Parati (Rio de Janeiro, Brasil). v. 52, n. 81, p. 49-64. 2001. il.

Leitão, Adriana Carrhá \& Silva, Oswaldo Aulino da. Variação sazonal de macronutrientes em uma espécie arbórea de cerrado, na Reserva Biológica e Estação Experimental de Mogi-Guaçu, estado de São Paulo, Brasil. v. 55, n. 84, p. 127-136. 2004.

Lemos, Jesus Rodrigues. Composição florística do Parque Nacional Serra da Capivara, Piauí, Brasil. v. 55, n. 85, p. 5566. 2004.

Lima, William Gomes \& Guedes-Bruni, Rejan R. Myrceugenia (Myrtaceae) ocorrentes no Parque Nacional do Itatiaia, Rio de Janeiro. v. 55, n. 85, p. 73-94. 2004. il.

Maas, Hiltje \& Maas, Paul J. M. Flora da Reserva Ducke, Amazonas, Brasil: Burmanniaceae. v. 56, n. 86, p. 125-130. 2005.

. Flora da Reserva Ducke, Amazonas, Brasil: Gentianaceae. v. 56, n. 86, p. 169173. 2005.

\& Maas, Hiltje. Flora da Reserva Ducke, Amazonas, Brasil: Triuridaceae. v. 56, n. 86, p. 209-211. 2005.

Maas, Paul J. M; Kamer, Hiltje Maas-van de; Junika, Leo; Mello-Silva, Renato de \& Rainer, Heimo. Annonnaceae from Central-eastern Brazil. v. 52, n. 80, p. 6194. 2001.

Maas, Paul \& Maas, Hiltje. Flora da Reserva Ducke, Amazonas, Brasil: Costaceae. v. 56, n. 86, p. 141-142. 2005.
Flora da Reserva Ducke, Amazonas, Brasil: Heliconiaceae. v. 56, n. 86, p. 175176. 2005.

. \& Maas, Hiltje. Flora da Reserva Ducke, Amazonas, Brasil: Strelitziaceae. v. 56, n. 86, p. 205. 2005.

. \& Maas, Hiltje. Flora da Reserva Ducke, Amazonas, Brasil: Thurniaceae. v. 56, n. 86, p. 207. 2005.

. \& Maas, Hiltje. Flora da Reserva Ducke, Amazonas, Brasil: Zingiberaceae. v. 56, n. 86, p. 213. 2005.

Mansano, Vidal de Freitas \& Tozzi, Ana Maria Goulart de Azevedo. Swartzia (Leguminosae, Papilonoideae, Swartzieneae s. l.) na Reserva Natural da Companhia Vale do Rio Doce, Linhares, ES, Brasil. v. 55, n. 85, p. 95-113. 2004. il.

Marques, Carlos Alexandre; Barros, Cláudia Franca \& Costa, Cecília Gonçalves. Beilschmiedia rigida (Mez) Kosterm. (Lauraceae): diferenciação e desenvolvimento da lâmina foliar. v. 55 , n. 84, p. 89100. 2004. il.

Marquete, Ronaldo. Reserva Ecológica do IBGE (Brasília-DF): Flacourtiaceae. v. 52, n. 80, p. 5-16. 2001. il.

Melo, José Iranildo Miranda de \& Sales, Margareth Ferreira de. Heliotropium L. (Boraginaceae - Heliotropioideae) de Pernambuco, Nordeste do Brasil. v. 55, n. 84, p. 65-87. 2004. il.

Mello-Silva, Renato de. Sistemática de Vellozia candida (Velloziaceae). v. 55, n. 84, p. 59-64. 2004. il.

Menini-Neto, L.; Almeida, V. R. \& Forzza, R. C. A família Orchidaceae na Reserva da Represa do Grama-Descoberto, Minas Gerais, Brasil. v. 55, n. 84, p. 137-156. 2004. il.

Molinaro, Lianna de Castro \& Costa, Denise Pinheiro da. Briófitas do arboreto do Jardim Botânico do Rio de Janeiro. v. 52, n. 81, p. 107-124. 2001.

Mynssen, Claudine M. \& Windisch, Paulo G. Pteridófitas da Reserva de Rio das 
Pedras, Mangaratiba, RJ, Brasil. v. 55, n. 85, p. 125-156. 2004. il.

Oliveira, Carlos Wagner de; Callado, Cátia Henriques \& Marquete, Osnir. Anatomia do lenho de espécies do gênero Nectandra Rol. ex Rottb. (Lauraceae). v. 52, n. 81, p. 125-134. 2001. il.

Oliveira, Rogério Ribeiro de. Ação antrópica e resultantes sobre a estrutura e composição da Mata Atlântica na Ilha Grande, RJ. v. 53, n. 82, p. 33-57. 2002.

Parente, Cláudio Ernesto.T. \& Rosa, Maria Mercedes.T. da. Plantas comercializadas como medicinais no município de Barra do Piraí, RJ. v. 52, n. 80, p. 47-59. 2001.

Parreiras, Oraida Maria Urbanetto de Souza. A regulamentação dos jardins botânicos brasileiros: ampliando as perspectivas de conservação da biodiversidade. v. 54, n. 83, p. 35-54. 2003.

Peixoto, Ariane Luna \& Escudeiro, Alexandra. Pachira aquatica (Bombacaceae) na obra "Historia dos Animais e Árvores do Maranhão" de Frei Cristóvão de Lisboa. v. 53, n. 82, p. 123-130. 2002. il.

Pirani, José Rubens. Flora da Reserva Ducke, Amazonas, Brasil: Rutaceae. v. 56, n. 86, p. 189-204. 2005. il.

Pivari, Marco Antonio; Forzza, Rafaela Campostrini. A família Palmae na Reserva Biológica da Represa do GramaDescoberto, Minas Gerias, Brasil. v. 55, n. 85 , p. $115-124$. 2004. il.

Prado, Jefferson. Flora da Reserva Ducke, Amazonas, Brasil: Pteridophyta - Chave para as famílias. v. 56 , n. 86 , p. 27-28. 2005.

. Flora da Reserva Ducke, Amazonas, Brasil: Pteridophyta - Aspleniaceae. v.56, n. 86, p.29-32. 2005. il.

Flora da Reserva Ducke, Amazonas, Brasil: Pteridophyta - Blechnaceae. v.56, n. 86, p.33-34. 2005. il.

Flora da Reserva Ducke, Amazonas, Brasil: Pteridophyta - Davalliaceae. v.56, n. 86, p. 38-42. 2005. il.
Flora da Reserva Ducke, Amazonas, Brasil: Pteridophyta-Dennstaedtiaceae. v. 56, n. 86, p. 43-48. 2005. il.

Flora da Reserva Ducke, Amazonas, Brasil: Pteridophyta - Gleicheniaceae. v. 56, n. 86, p. 53-55. 2005. il.

Flora da Reserva Ducke, Amazonas, Brasil: Pteridophyta - Grammitidaceae. v. 56, n. 86, p. 56-58. 2005. il.

. Flora da Reserva Ducke, Amazonas, Brasil: Pteridophyta-Lomariopsidaceae. v. 56, n. 86, p. 59-66. 2005. il.

Flora da Reserva Ducke, Amazonas, Brasil: Pteridophyta - Maratiaceae. v. 56, n. 86, p. 69-71. 2005. il.

Flora da Reserva Ducke, Amazonas, Brasil: Pteridophyta - Polypodiaceae. v. 56, n. 86, p. 76-84. 2005. il.

. Flora da Reserva Ducke, Amazonas, Brasil: Pteridophyta - Pteridaceae. v. 56, n. 86, p. 85-92. 2005.

Flora da Reserva Ducke, Amazonas, Brasil: Pteridophyta - Schizaeaceae. v. 56, n. 86, p. 93-97. 2005. il.

Flora da Reserva Ducke, Amazonas, Brasil: Pteridophyta - Tectariaceae. v. 56, n. 86, p. 103-104. 2005. il.

Flora da Reserva Ducke, Amazonas, Brasil: Pteridophyta - Thelypteridaceae. v. 56, n. 86, p.105-107. 2005. il.

Prado, Jefferson \& Freitas, Carlos, A A. Flora da Reserva Ducke, Amazonas, Brasil: Pteridophyta - Cyatheaceae. v. 56, n. 86, p. 35-37. 2005.

Prado, Jefferson \& Freitas, Carlos A. A. Flora da Reserva Ducke, Amazonas, Brasil: Pteridophyta - Selaginellaceae. v. 56, n. 86, p. 98-102. 2005. il.

Prado, Jefferson \& Labiak, Paulo H. Flora da Reserva Ducke, Amazonas, Brasil: Pteridophyta - Vittariaceae. v. 56, n. 86, p. 108-113. 2005. il.

Prance, Ghillean T. Flora da Reserva Ducke, Amazonas, Brasil: Rhabdodendraceae. v. 56, n. 86, p. 183-185. 2005. 
Flora da Reserva Ducke, Amazonas, Brasil: Rhizophoraceae. v. 56, n. 86, p. 187-188. 2005.

Quinet, Alexandre \& Andreata, Regina Helena Potsch. Lauraceae Jussieu na Reserva Ecológica de Macaé de Cima, Município de Nova Friburgo, Rio de Janeiro, Brasil. v. 53, n. 82, p. 59-121. 2002. il.

Sá, Cyl Farney Catarino de. Regeneração de um trecho de floresta de restinga na Reserva Ecológica Estadual de Jacarepiá, Saquarema, estado do Rio de Janeiro: II estrato arbustivo.v. 53, n. 82, p. 5-23. 2002. il.

Santos, Inês da Silva \& Peixoto, Ariane Luna. Taxonomia do gênero Macropeplus Perkins (Monimiaceae, Monimiodeae). v. 52, n. 81, p. 65-105. 2001.il.

Secco, Ricardo de S. Flora da Reserva Ducke, Amazonas, Brasil: Euphorbiaceae - Parte I. v. 56, n. 86. p. 143-168. 2005.

Silva, Marcos José da \& Sales, Margareth Ferreira de. O gênero Phyllanthus L. (Phyllantheae - Euphorbiaceae Juss.) no bioma Caatinga do estado de Pernambuco - Brasil. v. 55, n. 84, p. 101-126. 2004. il.
Silva, Nilda Marquete Ferreira da \& Valente, Maria da Conceição. Flora da Reserva Ducke, Amazonas, Brasil: Combretaceae. v. 56, n. 86, p. 131-140. 2005. il.

Valdéz, Adelaida Barreto; Carreras, Everardo Pérez; Artiles, Grisel Reyes; Salgueiro, Néstor Enríquez; Fariñas, Josefa Primelles \& Bueno, Erick Sedeño. Aportes al conocimiento de la riqueza florística para la gestión ambiental de la Sierra de Najasa, Camagüey, Cuba. v. 53, n. 82, p. 131145. 2002.

Vaz, Ângela M. Studart da Fonseca \& Tozzi, Ana Maria Goulart de Azevedo. Bauhinia ser. Cansenia (Leguminosae: Caesalpinoideae) no Brasil. v. 54, n. 83, p. 55-143. 2003. il.

Vieira, Cláudio M. \& Pessoa, Solange de V. A. Estrutura e composição florística do estrato herbáceo-subarbustivo de um pasto abandonado na Reserva Biológica de Poço das Antas, Município de Silva Jardim, RJ. v. 52, n. 80, p. 17-29. 2001.

Xavier, Sergio Romero da Silva \& Barros, Iva Carneiro Leão. Pteridófitas ocorrentes em fragmentos de floresta serrana no estado de Pernambuco, Brasil. v. 54, n. 83, p. 13-21. 2003.

Milton Ferreira Botelho*, Tânia Lúcia Rezende*, Maria de Fátima Verbicaro Ramos* \& Tânia Maura Nora Riccieri*

*Bibliotecários do Instituto de Pesquisas Jardim Botânico do Rio de Janeiro. Rua Jardim Botânico 1008, Rio de Janeiro, Brasil, CEP 22460-180. 